\title{
A new look at the pair-production width in a strong magnetic field
}

\author{
W. F. Kao, Guey-Lin Lin and Jie-Jun Tseng \\ Institute of Physics, National Chiao-Tung University, Hsinchu 300, Taiwan
}

(October 29, 2018)

\begin{abstract}
We reexamine the process $\gamma \rightarrow e^{+}+e^{-}$in a background magnetic field comparable to $B_{c} \equiv m_{e}^{2} / e$. This process is known to be non-perturbative in the magnetic-field strength. However, it can be shown that the moments of the above pair production width are proportional to the derivatives of photon polarization function at the zero energy, which is perturbative in $B$. Hence, the pairproduction width can be easily obtained from the latter by the inverse Mellin transform. The implications of our approach are discussed.
\end{abstract}

PACS numbers: 12.20.Ds, 11.55.Fv

The electroweak phenomena associated with an intensive background magnetic field are rather rich. Under a background magnetic field, a physical photon can decay into an $e^{+} e^{-}$pair or split into two photons. Such processes are relevant to the attenuation of gamma-rays from pulsars [1] 2]. The study of pair production process $\gamma+B \rightarrow e^{+} e^{-}+B$ was initiated by Toll [3] long time ago. He obtained a rather tedious expression for the absorption coefficient $\kappa_{\|, \perp}$, where $\|$ and $\perp$ denote the photon-polarization directions which are respectively parallel and perpendicular to the plane spanned by the magnetic field $\mathbf{B}$ and the photon momentum q. Writing

$$
\kappa_{\|, \perp}=\frac{\alpha}{2} \sin \theta\left(\frac{e B}{m_{e}}\right) T_{\|, \perp}(\lambda)
$$

with $\lambda=\frac{3}{2}\left(e B / m_{e}^{2}\right)\left(\omega / m_{e}\right) \sin \theta$, Toll obtained

$$
T_{\|, \perp}(\lambda)=\frac{9}{\lambda} \int_{(6 / \lambda)^{2 / 3}}^{\infty} d v\left[\frac{-\left(1-3 \eta_{\|, \perp} / 2 \lambda v^{3 / 2}\right) A^{\prime}(v)}{v^{5 / 4}\left(v^{3 / 2}-6 / \lambda\right)^{1 / 2}}+\frac{\left(v^{3 / 2}-6 / \lambda\right)^{1 / 2}}{3 v^{3 / 4}} A(v)\right],
$$

where $\eta_{\|}=1, \eta_{\perp}=3$ and

$$
A(v)=\frac{1}{2 \pi} \int_{-\infty+i \epsilon}^{\infty+i \epsilon} d t e^{i v t+i t^{3} / 3}
$$

where $\omega$ is the photon energy and $\theta$ is the angle between the magnetic-field direction and the direction of photon propagation. We note that, due to the quantization of electron and positron orbits in the magnetic field, $\kappa_{\|, \perp}$ should contain sawtooth absorption edges. However, for $B \ll B_{c} \equiv m_{e}^{2} / e$ and $\omega \sin \theta \gg 2 m_{e}$, these absorption edges are rather closely spaced. Hence it is sensible to define an averaged absorption coefficient which is precisely the $T_{\|, \perp}$ (with a trivial prefactor) displayed above. In other words, Toll's result is valid for $B \ll B_{c}$ and $\omega \sin \theta \gg 2 m_{e}$. It is interesting to note that, as pointed out by Toll, the functions $T_{\|, \perp}$ can not be calculated order by order in $e B$. It is essential to use the exact Dirac wave functions for electrons and positrons in the magnetic field such that the resulting $T_{\|, \perp}$ are nonvanishing. The non-analytic behaviors of $T_{\|, \perp}$ at $e B=0$ can be easily seen from its asymptotic expression for $\lambda \ll 1$ :

$$
T_{\|, \perp} \rightarrow \sqrt{\frac{3}{2}}\left(\frac{1}{2}, \frac{1}{4}\right) e^{-4 / \lambda}
$$

The expression for $T_{\|, \perp}$ was simplified considerably in the work by Tsai and Erber [5]. The authors computed the photon polarization function by the proper-time technique [6] and determined the absorption coefficient $\kappa_{\|, \perp}$ using the optical theorem. They obtained

$$
T_{\|, \perp}(\lambda)=\frac{4 \sqrt{3}}{\pi \lambda} \int_{0}^{1} d v\left(1-v^{2}\right)^{-1}\left[\left(1-\frac{1}{3} v^{2}\right),\left(\frac{1}{2}+\frac{1}{6} v^{2}\right)\right] K_{2 / 3}\left(\frac{4}{\lambda} \frac{1}{1-v^{2}}\right),
$$

where $K_{2 / 3}$ is the modified Bessel function. At the first glance, the result of Tsai and Erber appears very different from Toll's result. However, by computing the moments of $T_{\|, \perp}$, the former authors were able to show that their 
result is in fact equivalent to that of Toll]. We observe that Tsai and Erber simply utilized the moments of $T_{\|, \perp}$ as a mathematical tool to show the equivalence between two sets of absorption coefficients. The physical significance of these moments was not studied. In this note, we shall clarify the meaning of these moments and develop a new method of computing the absorption coefficients. Since our approach is essentially a systematic expansion in $B / B_{c}$, it will remain valid for a background magnetic field comparable to $B_{c}$.

We are motivated by the following contour integral, which resembles to the contour integral encountered in the QCD sum rule calculation of $e^{+} e^{-} \rightarrow$ hadrons [7]:

$$
I_{n}=\int_{C} \frac{d \omega^{2}}{2 \pi i} \frac{\Pi_{\|, \perp}\left(\omega^{2}\right)}{\left(\omega^{2}+\omega_{0}^{2}\right)^{n+1}},
$$

where the contour of integration $C$ is shown in Fig. 1.

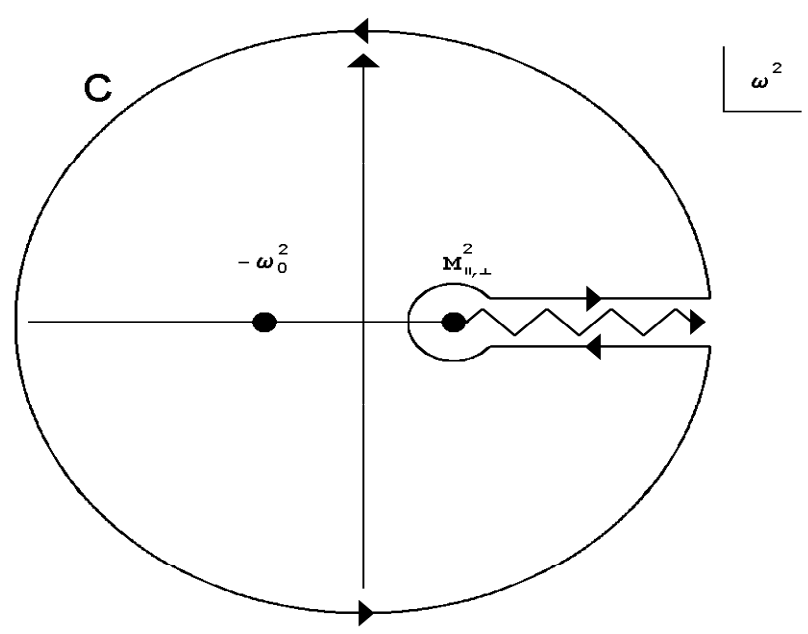

FIG. 1. The integration contour for $I_{n}$ and the analytic structure of $\Pi_{\|, \perp}$. In actual calculations, we take the radius of the circle to infinity.

The functions $\Pi_{\|, \perp}$ are defined as

$$
\Pi_{\|, \perp}=\epsilon_{\|, \perp}^{\mu} \Pi_{\mu \nu} \epsilon_{\|, \perp}^{\nu}
$$

where $\epsilon_{\|}^{\mu}$ and $\epsilon_{\perp}^{\mu}$ are respectively the photon polarization vectors parallel and perpendicular to the plane spanned by the photon momentum $\mathbf{q}$ and the magnetic field $\mathbf{B}$. We note that the integral $I_{n}$ may be evaluated in two different

\footnotetext{
${ }^{1}$ To state it more precisely, Tsai and Erber computed the moments of the averaged function $T \equiv 1 / 2 \cdot\left(T_{\|}+T_{\perp}\right)$, which is relevant to the attenuation of unpolarized photons.
} 
ways. One computes $I_{n}$ either by the residue theorem or by a direct integration along the contour $C$ with the realization that the contribution from the outer circle vanishes. The equivalence of two integration procedures gives rise to the relation:

$$
\left.\frac{1}{n !}\left(\frac{d^{n}}{d\left(\omega^{2}\right)^{n}} \Pi_{\|, \perp}\right)\right|_{\omega^{2}=-\omega_{0}^{2}}=\frac{1}{\pi} \int_{M_{\|, \perp}^{2}}^{\infty} d \omega^{2} \frac{\operatorname{Im} \Pi_{\|, \perp}\left(\omega^{2}\right)}{\left(\omega^{2}+\omega_{0}^{2}\right)^{n+1}}
$$

where $M_{\|, \perp}$ are the threshold energies of pair productions [3, [4] given by

$$
M_{\|}^{2} \sin ^{2} \theta=4 m_{e}^{2}, M_{\perp}^{2} \sin ^{2} \theta=m_{e}^{2}\left(1+\sqrt{1+2 \frac{B}{B_{c}}}\right)^{2},
$$

with $\theta$ the angle between the photon momentum and the magnetic field. Since $\kappa_{\|, \perp}=\operatorname{Im}_{\|, \perp} / \omega$ by the optical theorem, the above equation relate the real part of vacuum polarization function to the absorption coefficient.

We observe that the 1.h.s. of Eq. (8) can be easily calculated at $\omega^{2}=-\omega_{0}^{2}=0$, since the threshold behaviors of $\Pi_{\|, \perp}$ are absent at this energy value. With this choice of $\omega_{0}^{2}$, we recast Eq. (8) into

$$
\left.\frac{1}{n !}\left(\frac{d^{n}}{d\left(\omega^{2}\right)^{n}} \Pi_{\|, \perp}\right)\right|_{\omega^{2}=0}=\frac{M_{\|, \perp}^{1-2 n}}{\pi} \int_{0}^{1} d y \cdot y^{n-1} \cdot\left(\kappa_{\|, \perp}(y) y^{-1 / 2}\right) .
$$

with $y=M_{\|, \perp}^{2} / \omega^{2}$. One notes that the imaginary part of $\Pi_{\|, \perp}\left(\omega^{2}\right)$ vanishes for the range $0 \leq \omega^{2} \leq M_{\|, \perp}^{2}$. This property has been verified in the previous works [3, 4 . Therefore one can effectively set the integration range of Eq. (10) as from $y=0$ to $y=\infty$. Now, it is easily seen that the derivatives of $\Pi_{\|, \perp}$ at the zero energy are proportional to the Mellin transform of $\kappa_{\|, \perp} \cdot y^{-1 / 2} \equiv \kappa_{\|, \perp} \cdot \omega / M_{\|, \perp}$. Once the l.h.s. of Eq. (10) is calculated, the absorption coefficients $\kappa_{\|, \perp}$ can be determined by the inverse Mellin transform.

To calculate $\Pi_{\|, \perp}$ and their derivatives, we begin with the proper-time representation of vacuum polarization function $\Pi_{\mu \nu}$ in a background magnetic field [8]:

$$
\begin{aligned}
\Pi_{\mu \nu}(q) & =-\frac{e^{3} B}{(4 \pi)^{2}} \int_{0}^{\infty} d s \int_{-1}^{+1} d v\left\{e ^ { - i s \phi _ { 0 } } \left[\left(q^{2} g_{\mu \nu}-q_{\mu} q_{\nu}\right) N_{0}\right.\right. \\
& \left.-\left(q_{\|}^{2} g_{\| \mu \nu}-q_{\| \mu} q_{\| \nu}\right) N_{\|}+\left(q_{\perp}^{2} g_{\perp \mu \nu}-q_{\perp \mu} q_{\perp \nu}\right) N_{\perp}\right] \\
& \left.-e^{-i s m_{e}^{2}}\left(1-v^{2}\right)\left(q^{2} g_{\mu \nu}-q_{\mu} q_{\nu}\right)\right\}
\end{aligned}
$$

where

$$
\phi_{0}=m_{e}^{2}-\frac{1-v^{2}}{4} q_{\|}^{2}-\frac{\cos (z v)-\cos (z)}{2 z \sin (z)} q_{\perp}^{2}
$$

with $z=e B s$, and

$$
\begin{aligned}
& N_{0}=\frac{\cos (z v)-v \cot (z) \sin (z v)}{\sin (z)} \\
& N_{\|}=-\cot (z)\left(1-v^{2}+\frac{v \sin (z v)}{\sin (z)}\right)+\frac{\cos (z v)}{\sin (z)} \\
& N_{\perp}=-\frac{\cos (z v)}{\sin (z)}+\frac{v \cot (z) \sin (z v)}{\sin (z)}+2 \frac{\cos (z v)-\cos (z)}{\sin ^{3}(z)}
\end{aligned}
$$

To construct $\Pi_{\|, \perp}$ from $\Pi_{\mu \nu}$, we note that only the structures proportional to $N_{\|}$and $N_{\perp}$ contribute to $\Pi_{\|, \perp}$. Since we only concern with the limit $\omega \ll m_{e}$ and $B<B_{c}, \Pi_{\|, \perp}$ can be expanded in a series ${ }^{2}$ of $\omega$ and $B$ :

\footnotetext{
${ }^{2}$ In fact, we do not need Eq. (11) to obtain such an expansion. A convenient weak-field expansion technique applicable to the current problem has been developed in Ref. 9].
} 


$$
\Pi_{\|, \perp}=\sum_{n=1}^{\infty} \frac{2 \alpha m_{e}^{2}}{\pi}\left(\frac{\omega^{2} \sin ^{2} \theta B^{2}}{3 m_{e}^{2} B_{c}^{2}}\right)^{n} \frac{\Gamma(3 n-1) \Gamma^{2}(2 n)}{\Gamma(n) \Gamma(4 n)}\left(\frac{6 n+1,3 n+1}{4 n+1}\right)+\cdots
$$

where the neglected terms are of the order $\left(\omega^{2} B^{2} \sin ^{2} \theta / m_{e}^{2} B_{c}^{2}\right)^{n}\left(B / B_{c}\right)^{2}$. Taking the derivatives of $\Pi_{\|, \perp}$, we arrive at

$$
\left.\frac{1}{n !}\left(\frac{d^{n}}{d\left(\omega^{2}\right)^{n}} \Pi_{\|, \perp}\right)\right|_{\omega^{2}=0}=\frac{2 \alpha m_{e}^{2}}{\pi}\left(\frac{B^{2} \sin ^{2} \theta}{3 B_{c}^{2} m_{e}^{2}}\right)^{n} \frac{\Gamma(3 n-1) \Gamma^{2}(2 n)}{\Gamma(n) \Gamma(4 n)}\left(\frac{6 n+1,3 n+1}{4 n+1}\right)+\cdots
$$

Combining the above equation and Eq. (10), the absorption coefficients $\kappa_{\|, \perp}$ can be written in terms of inverse Mellin transform:

$$
\begin{aligned}
\kappa_{\|} & =\frac{\alpha m_{e}^{2}}{i \pi \omega} \int_{-i \infty+a}^{+i \infty+a} d s\left(\lambda^{\prime}\right)^{2 s} \frac{\Gamma(3 s) \Gamma^{2}(2 s)}{\Gamma(s) \Gamma(4 s)} \frac{1}{3 s-1} \times \frac{6 s+1}{4 s+1} \\
\kappa_{\perp} & =\frac{2 \alpha m_{e}^{2}}{i \pi \omega} \frac{1}{1+\sqrt{1+2 B / B_{c}}} \int_{-i \infty+a}^{+i \infty+a} d s\left(\lambda^{\prime \prime}\right) \frac{2 s(3 s) \Gamma^{2}(2 s)}{\Gamma(s) \Gamma(4 s)} \frac{1}{3 s-1} \times \frac{3 s+1}{4 s+1},
\end{aligned}
$$

where $a$ is any real number greater than $1 / 3$; while $\lambda^{\prime}=\left(\omega \sin \theta B / \sqrt{3} m_{e} B_{c}\right)$ and $\lambda^{\prime \prime}=\lambda^{\prime} \cdot\left(1+\sqrt{1+2 B / B_{c}}\right) / 2$. At this moment, we only concern with the leading magnetic-field effect to the absorption coefficients, hence we may set $\left(1+\sqrt{1+2 B / B_{c}}\right) \rightarrow 2$ and $\lambda^{\prime \prime} \rightarrow \lambda^{\prime}$ in $\kappa_{\perp}$. Numerically we find no distinctions between our results and the results of Tsai and Erber [5]. For $\lambda^{\prime}=10$, we have $\kappa_{\|}=7.2 \times\left(\alpha m_{e}^{2} / \pi \omega\right)$ while $\kappa_{\perp}=4.4 \times\left(\alpha m_{e}^{2} / \pi \omega\right)$. For $\lambda^{\prime}=100$, the above absorption coefficients become $42 \times\left(\alpha m_{e}^{2} / \pi \omega\right)$ and $28 \times\left(\alpha m_{e}^{2} / \pi \omega\right)$ respectively. In the current approximation, $\kappa_{\|}$is always greater than $\kappa_{\perp}$. For a high-energy photon, i.e., $\lambda^{\prime} \gg 1$, we have $\kappa_{\|} / \kappa_{\perp}=1.5$. This is already reflected in the above case with $\lambda^{\prime}=100$. For the low-energy photon, $\lambda^{\prime} \ll 1$, we find $\kappa_{\|} / \kappa_{\perp}=2$. The numerical agreement between Eq. (16) and the result of Ref. [5], as shown in Eq. (5), is not a coincidence. We shall verify shortly that both expressions are equivalent by comparing their infinite sequences of moments.

As mentioned earlier, in order to establish the equivalence of their result with those of the previous works, the authors of Ref. [5] computed the moments $C_{n}^{(1)}=\int_{0}^{\infty} d \chi \chi^{n} T(\chi)$ with $T(\chi)=1 / 2 \cdot\left(T_{\|}(\chi)+T_{\perp}(\chi)\right)$ and $\chi=\left(4 m_{e} B_{c} / 3 \omega B\right)$. The superscript (1) is used to denote the set of moments computed from the functions $T_{\|, \perp}$ given in Ref. [5]. The superscript (0) will then be used for denoting the moments computed from our results for $T_{\|, \perp}$. Without taking the average, we obtain the moments of each individual function $T_{\|}$and $T_{\perp}$, which we denote as $C_{n}^{(1)}(\|)$ and $C_{n}^{(1)}(\perp)$ respectively. We obtain

$$
C_{n}^{(1)}(\|, \perp)=2^{-2}(3 / \pi)^{1 / 2} \frac{(6 n+14,3 n+8)}{2 n+5} \frac{\Gamma(n / 2+2 / 3) \Gamma(n / 2+4 / 3) \Gamma(n+2)}{\Gamma(n+5 / 2)},
$$

To compare $C_{n}^{(1)}(\|, \perp)$ with the moments pertinent to Eq. (10), i.e., $D_{n}^{(0)} \equiv \int_{0}^{\infty} d y \cdot y^{n-1} \cdot\left(T_{\|, \perp}(y) y^{-1 / 2}\right)$, we employ the relation

$$
D_{n}^{(0)}(\|, \perp)=2\left(\frac{3 B}{2 B_{c} \sin \theta}\right)^{2 n-1} C_{2 n-2}^{(0)}(\|, \perp)
$$

where $C_{2 n-2}^{(0)}(\|, \perp)$ is defined in the same way as $C_{2 n-2}^{(1)}(\|, \perp)$. Our first goal is to show that $C_{2 n-2}^{(0)}(\|, \perp)=C_{2 n-2}^{(1)}(\|, \perp)$. In fact, this identity can be established by combining the relation

$$
D_{n}^{(0)}(\|, \perp)=\frac{2^{2 n+1}}{3^{n}}\left(\frac{B}{B_{c}}\right)^{2 n-1} \frac{\Gamma(3 n-1) \Gamma(2 n) \Gamma(2 n)}{\Gamma(n) \Gamma(4 n)} \cdot \frac{(6 n+1,3 n+1)}{4 n+1},
$$

derived from Eqs. (10) and (15), with Eqs. (17), (18) and the identity

$$
\frac{\Gamma(n-1 / 3) \Gamma(n+1 / 3)}{\Gamma(2 n+1 / 2)}=\frac{2^{4 n} \sqrt{\pi}}{3^{3 n-3 / 2}} \times \frac{\Gamma(3 n-1) \Gamma(2 n)}{\Gamma(n) \Gamma(4 n)} .
$$

Now that we have shown $C_{2 n-2}^{(0)}(\|, \perp)=C_{2 n-2}^{(1)}(\|, \perp)$, we obtain the identity $D_{n}^{(0)}(\|, \perp)=D_{n}^{(1)}(\|, \perp)$ where $D_{n}^{(1)}(\|, \perp)$ is given by the r.h.s. of Eq. (18) with $C_{2 n-2}^{(0)}(\|, \perp)$ replaced by $C_{2 n-2}^{(1)}(\|, \perp)$. Since both $D_{n}$ 's are identical, one can 
show that the absorption coefficient derived from our approach, Eq. (16), is equivalent to the result of Ref. [5] given by Eqs. (5) and (1), provided that

$$
\sum_{n} D_{n}^{-1 / 2 n} \rightarrow \infty
$$

according to the Carleman's theorem [10]. Indeed, this is true since

$$
\sum_{n} D_{n}^{-1 / 2 n} \rightarrow \sum_{n} \frac{1}{n} \rightarrow \infty \text { as } D_{n} \sim \frac{9}{8}\left(\frac{2 \pi}{3}\right)^{1 / 2} n^{2 n-3 / 2} e^{2-2 n}
$$

Therefore, we have proven that the leading-order results of our approach agree with the results of Ref. [5]. In addition, as one can see from the r.h.s. of Eq. (18), only the even moments defined by Tsai and Erber are relevant to the physics of pair-production in a background magnetic field.

We like to point out the differences between our approach and the approach of Ref. [5]. Tsai and Erber begin with $\Pi_{\mu \nu}$ given in Eq. (11) and evaluate the imaginary part of $\Pi_{\mu \nu}$ for $\omega \equiv q^{0}$ greater than the pair production threshold. They arrive at the asymptotic result, Eq. (5), in the limit $B \ll B_{c}$ and $\omega \sin \theta \gg 2 m_{e}$. However, their approach does not provide an estimate of possible corrections as $B$ and $\omega$ deviate from the above limit. Our approach has an advantage in that it treats the magnetic-field effects perturbatively for $B<B_{c}$. In Eq. (15), the $n$-th derivative of the vacuum-polarization function $\Pi_{\|, \perp}$ is expanded in powers of $B^{2} / B_{c}^{2}$. Hence the absorption coefficient, which is related to the derivatives of $\Pi_{\|, \perp}$ by an inverse Mellin transform, can also be written in powers of $B^{2} / B_{c}^{2}$. In this way, we are able to compute the absorption coefficient even for $B$ comparable to $B_{c}$. As for the low energy regime near the pair production threshold, $\omega \sin \theta \gtrsim 2 m_{e}$, the quantum effects due to the magnetic field become important. Namely, for given $\omega$ and $B$, the momenta of $e^{+}$and $e^{-}$along the magnetic-field direction can only take discrete values, and consequently the absorption coefficients $\kappa_{\|, \perp}$ contain resonant peaks. The spacing of these peaks increases as $\omega \sin \theta$ gradually decreases to the pair-production threshold $2 m_{e}$. A detailed study of this threshold behavior has been initiated by Daugherty and Harding [11]. In Fig. 6 of Ref. [11], it is shown that the threshold behavior is non-negligible for $\xi \equiv \omega^{2} B_{c} / 2 m_{e}^{2} B<10^{3}$ with $\sin ^{2} \theta=1$. For a general $\theta$, the relevant parameter becomes $\xi^{\prime}=\omega^{2} \sin ^{2} \theta B_{c} / 2 m_{e}^{2} B$. It should be understood that our result as well as the result of Ref. [5] are applicable for a large $\xi\left(\xi^{\prime}\right)$ where the threshold effect is not significant.

We have mentioned that our results for $\kappa_{\|, \perp}$ are written as power series in $\left(B / B_{c}\right)^{2}$. It is important to compute the next-to-leading corrections. Let us begin by computing the next-to-leading magnetic-field corrections to $\Pi_{\|}$and its derivatives. To do this we perform a weak-field expansion with respect to the exponent $\phi_{0}$ and the amplitude $N_{\|}$, along with a rotation of the integration contour $s \rightarrow-i s$ :

$$
\begin{aligned}
\phi_{0} & =m_{e}^{2}-\frac{\omega^{2} \sin ^{2} \theta}{48} z^{2}\left(1-v^{2}\right)^{2}\left(1-\frac{1}{30}\left(3-v^{2}\right) z^{2}+\cdots\right), \\
-i N_{\|} & \rightarrow\left[\frac{\cosh (z v)}{\sinh (z)}-\frac{\cosh (z)}{\sinh (z)}\left(1-v^{2}+\frac{v \sinh (z v)}{\sinh (z)}\right)\right] \\
& =-\frac{z}{6}\left(1-v^{2}\right)\left(3-v^{2}\right)+\frac{z^{3}}{360}\left(1-v^{2}\right)\left(15-2 v^{2}+3 v^{4}\right)+\cdots
\end{aligned}
$$

Hence the next-to-leading (NL) corrections to the derivatives of $\Pi_{\|}$reads:

$$
\begin{aligned}
\left.\frac{1}{n !}\left(\frac{d^{n}}{d\left(\omega^{2}\right)^{n}} \Pi_{\|}^{N L}\right)\right|_{\omega^{2}=0} & =\frac{-2 \alpha m_{e}^{2}}{5 \pi}\left(\frac{B}{B_{c}}\right)^{2}\left(\frac{B^{2} \sin ^{2} \theta}{3 B_{c}^{2} m_{e}^{2}}\right)^{n} \frac{n \Gamma(3 n) \Gamma^{2}(2 n)}{\Gamma(n) \Gamma(4 n)} \\
& \times \frac{\left(3+2 n+24 n^{2}+36 n^{3}\right)}{(4 n+1)(4 n+3)} .
\end{aligned}
$$

Using Eq. (10), and applying the inverse Mellin transform, we arrive at

$$
\kappa_{\|}^{N L}=\frac{-\alpha m_{e}^{2}}{10 i \pi \omega}\left(\frac{B}{B_{c}}\right)^{2} \int_{-i \infty+b}^{+i \infty+b} d s\left(\lambda^{\prime}\right)^{2 s} \frac{\Gamma(3 s) \Gamma^{2}(2 s) s}{\Gamma(s) \Gamma(4 s)} \times \frac{\left(3+2 s+24 s^{2}+36 s^{3}\right)}{(4 s+1)(4 s+3)},
$$

where $b$ can be chosen to be any positive number. Numerically, for $\left(B / B_{c}\right)^{2}=0.1$ and $\lambda^{\prime}=10$, we have $\kappa_{\|}^{N L}=-1.5 \times$ $10^{-3} \times\left(\alpha m_{e}^{2} / \pi \omega\right)$. We note that $\xi^{\prime} \approx 10^{4}$ for the current values of $B$ and $\lambda^{\prime}$. In this case $\left|\kappa_{\|}^{N L} / \kappa_{\|}\right|<0.1 \%$. For the same 
magnetic-field strength with $\xi^{\prime}=10^{3}\left(\lambda^{\prime} \approx 3\right)$, we obtained $\kappa_{\|}=2.0 \times\left(\alpha m_{e}^{2} / \pi \omega\right)$ and $\kappa_{\|}^{N L}=7.7 \times 10^{-4} \times\left(\alpha m_{e}^{2} / \pi \omega\right)$. The ratio $r \equiv\left|\kappa_{\|}^{N L} / \kappa_{\|}\right|$remains to be less than $0.1 \%$ in this case. Hence in the energy regime that the quantum effects of the magnetic field is not essential, the subleading contribution to the absorption coefficient, $\kappa_{\|}^{N L}$, is rather suppressed. If we extrapolate our analysis down to the energy of pair-production threshold $\omega \sin \theta=2 m_{e}$ while maintaining $\left(B / B_{c}\right)^{2}=0.1$, i.e., $\lambda^{\prime}=0.35$, we find $\kappa_{\|}=6.5 \times 10^{-3} \times\left(\alpha m_{e}^{2} / \pi \omega\right)$ and $\kappa_{\|}^{N L}=-2.2 \times 10^{-3} \times\left(\alpha m_{e}^{2} / \pi \omega\right)$. It is interesting to see that $\kappa_{\|}^{N L}$ is of the same order of magnitude as the leading contribution. This reflects the limitation of our approach and that of Tsai and Erber near the pair-production threshold.

The next-to-leading correction to $\kappa_{\perp}$ is calculated in a similar way. The expansion of $\phi_{0}$ proceeds as before while

$$
\begin{aligned}
-i N_{\perp} & \rightarrow\left[-\frac{\cosh (z v)}{\sinh (z)}+\frac{v \cosh (z) \sinh (z v)}{\sinh ^{2}(z)}-\frac{2(\cosh (z v)-\cosh (z))}{\sinh ^{3}(z)}\right] \\
& =-\frac{z}{12}\left(1-v^{2}\right)\left(3+v^{2}\right)+\frac{z^{3}}{180}\left(1-v^{2}\right)\left(15-6 v^{2}-v^{4}\right)+\cdots .
\end{aligned}
$$

Then the next-to-leading corrections to the derivatives of $\Pi_{\perp}$ are

$$
\begin{aligned}
\left.\frac{1}{n !}\left(\frac{d^{n}}{d\left(\omega^{2}\right)^{n}} \Pi_{\perp}^{N L}\right)\right|_{\omega^{2}=0} & =\frac{-2 \alpha m_{e}^{2}}{5 \pi}\left(\frac{B}{B_{c}}\right)^{2}\left(\frac{B^{2} \sin ^{2} \theta}{3 B_{c}^{2} m_{e}^{2}}\right)^{n} \frac{n \Gamma(3 n) \Gamma^{2}(2 n)}{\Gamma(n) \Gamma(4 n)} \\
& \times \frac{\left(3+39 n+60 n^{2}+18 n^{3}\right)}{(4 n+1)(4 n+3)} .
\end{aligned}
$$

Applying the inverse Mellin transform, we obtain

$$
\kappa_{\perp}^{N L}=\frac{-\alpha m_{e}^{2}}{10 i \pi \omega}\left(\frac{B}{B_{c}}\right)^{2} \int_{-i \infty+c}^{+i \infty+c} d s\left(\lambda^{\prime}\right)^{2 s} \frac{\Gamma(3 s) \Gamma^{2}(2 s) s}{\Gamma(s) \Gamma(4 s)} \times \frac{\left(3+39 s+60 s^{2}+18 s^{3}\right)}{(4 s+1)(4 s+3)},
$$

where $c$ can be chosen as any positive number, and, to isolate the $O\left(B^{2} / B_{c}^{2}\right)$ corrections, we have made the identifications $\lambda^{\prime \prime} \rightarrow \lambda^{\prime}$ and $\left(1+\sqrt{1+2 B / B_{c}}\right) \rightarrow 2$. Numerically, for $\left(B / B_{c}\right)^{2}=0.1$ and $\lambda^{\prime}=10$, we have $\kappa_{\perp}=4.4 \times\left(\alpha m_{e}^{2} / \pi \omega\right)$ while $\kappa_{\|}^{N L}=1.3 \times 10^{-3} \times\left(\alpha m_{e}^{2} / \pi \omega\right)$. Similar to the $\kappa_{\|}$case, $\left|\kappa_{\perp}^{N L} / \kappa_{\perp}\right|<0.1 \%$ for $\lambda^{\prime}=10$. For $\lambda^{\prime}=3$ which corresponds to $\xi^{\prime} \approx 10^{3}$, we find $\kappa_{\perp}=1.2 \times\left(\alpha m_{e}^{2} / \pi \omega\right)$ and $\kappa_{\perp}^{N L}=-4 \times 10^{-3} \times\left(\alpha m_{e}^{2} / \pi \omega\right)$. In this case $\left|\kappa_{\perp}^{N L} / \kappa_{\perp}\right| \approx 0.3 \%$. We observe again that the ratio, $\left|\kappa_{\perp}^{N L} / \kappa_{\perp}\right|$, grows rapidly to $60 \%$ at the energy of pair-production threshold with $\kappa_{\perp}^{N L}$ being negative.

¿From the above next-to-leading order calculations, it is quite evident that the $O\left(B^{2} / B_{c}^{2}\right)$-corrections to $\kappa_{\|}$and $\kappa_{\perp}$ are both rather insignificant. However, one should be reminded that there are still $O\left(B / B_{c}\right)$ - corrections to $\kappa_{\perp}$ as shown in Eq.(16). Without making the identifications $\lambda^{\prime \prime} \rightarrow \lambda^{\prime}$ and $\left(1+\sqrt{1+2 B / B_{c}}\right) \rightarrow 2$, we have $\kappa_{\perp}=3.8 \times$ $\left(\alpha m_{e}^{2} / \pi \omega\right)$ for $\lambda^{\prime \prime}=10 \times\left(1+\sqrt{1+2 B / B_{c}}\right) / 2$ (i.e., $\left.\lambda^{\prime}=10\right)$ with $\left(B / B_{c}\right)^{2}=0.1$. We recall that $\kappa_{\perp}=4.4 \times\left(\alpha m_{e}^{2} / \pi \omega\right)$ if the above identifications are made. Hence the $O\left(B / B_{c}\right)$-correction reduces $\kappa_{\perp}$ by about $14 \%$ at the current $\omega$ and $B$. Essentially, the $O\left(B / B_{c}\right)$-corrections to $\kappa_{\perp}$ relative to $\kappa_{\|}$are kinematic in nature. They are due to the differences in pair-production threshold resulting from different polarization states of the decaying photons. For a photon with the $\|$-polarization, the pair-production threshold corresponds to both $e^{+}$and $e^{-}$being in the ground state. On the other hand, for a photon with the $\perp$-polarization, either $e^{+}$or $e^{-}$must be in the first excited state at the pair-production threshold [3].

Since the $O\left(B^{2} / B_{c}^{2}\right)$-corrections to $\kappa_{\|, \perp}$ can be neglected, Eq. (16) are accurate expressions for photon absorption coefficients even to a magnetic-field strength comparable to $B_{c}$. In Fig. 2, we plot $\kappa_{\|, \perp}$ as functions of $\omega$ with $\sin ^{2} \theta=1$ and $\left(B / B_{c}\right)^{2}=0.1$. Here our result for $\kappa_{\|}$is identical to that of Tsai and Erber, whereas our result for $\kappa_{\perp}$ contains the kinematic corrections which were not taken into accounts in previous works. As can be seen from Fig. 2, both $\kappa_{\|}$and $\kappa_{\perp}$ are rather insensitive to $\omega$ once $\omega$ surpasses $10 \mathrm{MeV}$. Hence, for a sufficiently large $\omega$, the kinematic corrections to $\kappa_{\perp}$ mainly reside in the factor $1 /\left(1+\sqrt{1+2 B / B_{c}}\right)$ rather than in $\lambda^{\prime \prime}$. It should be noted that the applicability of our result is determined by the parameter $\xi^{\prime}=\omega^{2} \sin ^{2} \theta B_{c} / 2 m_{e}^{2} B$ [11]. For the parameter set in Fig. 2, i.e., $\left(B / B_{c}\right)^{2}=0.1$ and $\sin ^{2} \theta=1$, we have $\omega \approx 25 m_{e}=12.5 \mathrm{MeV}$ for $\xi^{\prime}=10^{3}$. Hence, one expects the plots in Fig. 2 to be reliable for $\omega>10 \mathrm{MeV}$. 


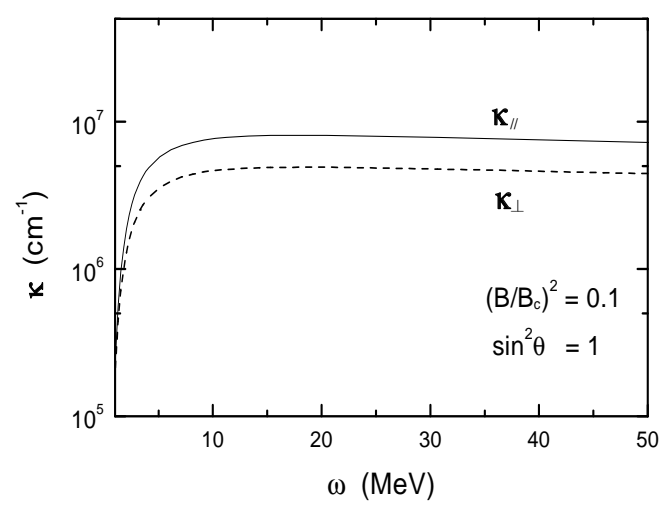

FIG. 2. The photon absorption coefficients as functions of the photon energy with $\sin ^{2} \theta=1$ and $\left(B / B_{c}\right)^{2}=0.1$.

At this point, one might conclude that our approach can not describe the threshold behavior of the absorption coefficient. This is in fact not true. We should stress that it is the weak-field expansion of Eq. (15) that spoils the threshold behavior of the absorption coefficient, despite such an expansion is useful for computing the absorption coefficient at much higher energies. To see this, it is instructive to review how Eq. (15) is derived. In this note, we obtained Eq. (15) by expanding Eq. (11) directly. However, one could also derive Eq. (15) by expanding the internal fermion propagators in $\Pi_{\|, \perp}$ in powers of $e B$. This expansion has been derived in Eq. (47) of Ref. [9]. In that equation, one can see that the weak-field expansion mixes contributions coming from different Landau levels. Hence the threshold behaviors of $\Pi_{\|, \perp}$ at any given Landau level are spoiled by the weak-field expansion. In fact, the absorption coefficients $\kappa_{\|, \perp}$ calculated from $\Pi_{\|, \perp}$ do not vanish below the pair-production threshold, i.e., $0 \leq \omega^{2} \leq M_{\|, \perp}^{2}$. This can be seen by performing the integration in Eq. (16). Such a behavior is again an artifact caused by the weak field expansion performed near the pair-production threshold. Indeed, as mentioned before, $\kappa_{\|, \perp}^{N L}$ are comparable to $\kappa_{\|, \perp}$ with an opposite sign in this energy range. The cancellation of these two contributions is consistent with the fact that the absorption coefficients should vanish below the pair-production threshold [3. 4 .

To recover the threshold behavior, one may compute $\Pi_{\mu \nu}$ using electron propagators in the Furry picture [12,13]:

$$
S_{F}^{B}\left(x^{\prime}, x\right)_{\alpha \beta}=\sum_{n=0}^{\infty} \int \frac{d \omega d q^{2} d q^{3}}{(2 \pi)^{3}} \frac{\exp \left(-i \omega\left(t^{\prime}-t\right)+i q^{2}\left(x^{\prime 2}-x^{2}\right)+i q^{3}\left(x^{\prime 3}-x^{3}\right)\right)}{q_{\|}^{2}-m_{e}^{2}-2 n e B+i \epsilon}\left(S_{n ; \omega, q^{2}, q^{3}}\right)_{\alpha \beta},
$$

where $q_{\|}^{2}=\left(q^{0}\right)^{2}-\left(q^{3}\right)^{2}, n$ is the quantum number for the Landau level, and $S_{n ; \omega, q^{2}, q^{3}}$ is a $4 \times 4$ matrix in the spinor space. In this form, all the poles of the propagator appear explicitly, and the threshold behavior of $\Pi_{\mu \nu}$ is preserved throughout the calculation [14]. Clearly, a calculation using the Furry-picture propagators compliments the weak-field expansion technique we have been discussing so far. The former produces a correct threshold-behavior of the absorption coefficient but becomes unpractical at larger energies, since, in such a case, contributions from a great number of Landau levels has to be summed over. Nevertheless, in the scenario that $B \gg B_{c}$, it is convenient to use the Furry-picture propagators because the available Landau levels for pair production to occur are significantly reduced.

In conclusion, we have developed a new method for computing the photon absorption coefficient in a strong background magnetic field $B \lesssim B_{c}$. Disregarding the next-to-leading magnetic-field corrections, our $\kappa_{\|}$is identical to that obtained by Tsai and Erber [5]. Although Tsai and Erber derived $\kappa_{\|}$under the assumption $B \ll B_{c} \equiv m_{e}^{2} / e$ and $\omega \sin \theta \gg 2 m_{e}$, we have been able to show that such a result is in fact accurate for $B$ comparable to $B_{c}$, provided $\omega$ $\left(\xi^{\prime}\right)$ is large enough. For $\kappa_{\perp}$, our result differs from that obtained in Ref. [5]. In this regard, we have identified certain $O\left(B / B_{c}\right)$ corrections to $\kappa_{\perp}$ which are kinematic in nature. We also pointed out that our approach may be extended to lower photon energies near the pair-production threshold, so long as we calculate $\Pi_{\mu \nu}$ with electron propagators in the Furry picture [13]. For a supercritical magnetic field $B \gg B_{c}$, we argued that it is convenient to use the Furry-picture propagators. As a closing, we like to emphasize that a better understanding of the current process is crucial for determining the photon attenuation properties in highly magnetized pulsars [15]. 


\section{ACKNOWLEDGMENTS}

We thank H.-K. Chang for bringing Ref. [11] to our attentions. This work is supported in part by the National Science Council under grant numbers NSC89-2112-M009-001 and NSC89-2112-M009-035.

[1] P. A. Sturrock, ApJ 164, 529, 1971.

[2] M. G. Baring and A. K. Harding, ApJ 482, 372, 1997.

[3] J. S. Toll, Ph.D. thesis, Princeton Univ., 1952 (unpublished).

[4] S. L. Adler, Ann. Phys. (N.Y.) 67, 599, 1971.

[5] W.-y. Tsai and T. Erber, Phys. Rev. D 10, 492, 1974.

[6] J. Schwinger, Phys. Rev. 82, 664, 1951.

[7] M. A. Shifman, A. I. Vainstein, and V. I. Zakharov, Nucl. Phys. B 147, 385, 1979.

[8] W.-y. Tsai, Phys. Rev. D 10, 2699, 1974.

[9] T.-K. Chyi, C.-W. Hwang, W.-F. Kao, G.-L. Lin, K.-W. Ng and J.-J. Tseng, hep-th/9912134, to appear in Phys. Rev. D.

[10] T. Carleman, Les fonctions quasi-analytiques (Herman, Paris, 1926).

[11] J. K. Daugherty and A. K. Harding, ApJ 273, 761, 1983.

[12] W. H. Furry, Phys. Rev. 81, 115, 1951.

[13] M. Kobayashi and M. Sakamoto, Prog. Theor. Phys., 70, 1375, 1983.

[14] W.-F. Kao, G.-L. Lin and J.-J. Tseng, work in progress.

[15] M. G. Baring and A. K. Harding, ApJ 507, L55, 1998. 